\title{
Diffuse Alveolar Hemorrhage Associated with Warfarin Therapy
}

\author{
Bülent Kaya, ${ }^{1}$ Ibrahim Yildiz, ${ }^{2}$ Reshat Mehmet Baha, ${ }^{2}$ \\ Neslihan Ebru Eryaşar Zeytun, ${ }^{2}$ and Azize Yetisgen ${ }^{3}$ \\ ${ }^{1}$ Nephrology Department, Osmaniye State Hospital, Turkey \\ ${ }^{2}$ Cardiology Department, Osmaniye State Hospital, Turkey \\ ${ }^{3}$ Infectious Diseases Department, Osmaniye State Hospital, Turkey \\ Correspondence should be addressed to Bülent Kaya; bulentkaya32@gmail.com
}

Received 13 February 2015; Revised 18 June 2015; Accepted 21 June 2015

Academic Editor: W. Zidek

Copyright (C) 2015 Bülent Kaya et al. This is an open access article distributed under the Creative Commons Attribution License, which permits unrestricted use, distribution, and reproduction in any medium, provided the original work is properly cited.

Diffuse alveolar hemorrhage (DAH) is a life-threatening clinical pathologic syndrome caused by a variety of diseases. We report a case of DAH related to therapy of warfarin use. In this case report, we present the diffuse alveolar hemorrhage case as a rare and life-threatening complication of warfarin.

\section{Introduction}

Diffuse alveolar hemorrhage (DAH) is a life-threatening clinical pathologic syndrome caused by a variety of diseases. We report a case of DAH related to therapy of warfarin use. DAH due to warfarin therapy has been rarely reported in the literature. In this paper, we describe the case of a 59-yearold man with a history of mitral valve replacement (MVR) hypertension (HT), diabetes mellitus (DM), and chronic renal failure $(\mathrm{CRF})$ treated with warfarin, who was admitted to our institution with haemoptysis and dyspnea. Alveolar hemorrhage was suspected clinically and radiologic findings were subsequently confirmed by bronchoscopy. The patient was successfully treated with 6 units of fresh frozen plasma (FFP), 4 units of red blood cell (RBC) transfusion, and hemodialysis.

\section{Case Presentation}

A 59-year-old man with a past medical history of HT, DM, MVR, and CRF was admitted to our institution complaining of haemoptysis and shortness of breath over the previous $24 \mathrm{~h}$.

On examination, he was noted to be in mild respiratory distress and was coughing up blood-tinged sputum. His vital signs were temperature $37,4^{\circ} \mathrm{C}$, pulse $106 \mathrm{bpm}$, and regular, respiratory rate 20 per minute, blood pressure $140 / 77 \mathrm{~mm} \mathrm{Hg}$, and oxygen saturation $94 \%$ on room air. He had rhythmic metallic first heart sound and a normal second heart sound. He also had diffuse wet crackles over both lung fields. The rest of his physical examination was unremarkable.

The patient's medications on the day of admission were amlodipine $10 \mathrm{mg}$ PO daily, warfarin 2,5 mg PO daily, and carvedilol 12,5 mg PO daily.

The patient's laboratory findings were WBC 8,8 haemoglobin $7,5 \mathrm{~g} / \mathrm{dL}$, haematocrit $22,3 \%$, platelets 148,000 , INR 7,9, PT $94 \mathrm{~s}$, a PTT $120 \mathrm{~s}$, random glucose $177 \mathrm{mg} / \mathrm{dL}$, serum sodium $141 \mathrm{mmol} / \mathrm{L}$, serum potassium $4 \mathrm{mmol} / \mathrm{L}$, and serum creatinine $8,6 \mathrm{mg} / \mathrm{dL}$. Urinalysis showed very high number of RBCs without RBC casts. The rest of the laboratory results, including D-dimer level and cardiac enzymes, were unremarkable.

The patient was started empirically on moxifloxacin for possible community acquired pneumonia. Serum levels of C-ANCA, P-ANCA, ANA, and anti-ds-DNA were negative. Transthoracic echocardiography showed left ventricular ejection fraction of 0.45 and a normal mechanical mitral valve prosthesis with normal gradient. Abdominal ultrasound showed bilateral normal kidneys and bilaterally increased renal cortical echogenicity.

Chest radiographs revealed that the lungs were bilaterally infiltrated (Figure 1). A high resolution computed tomographic study of the thorax disclosed DAH (Figure 2), the presence of which was proved by bronchoscopy. 
TABLE 1: The patient's labarotary parameters, according to date and levels.

\begin{tabular}{lcccccccccccc}
\hline Date & 10.1 .15 & 11.1 .15 & 12.1 .15 & 14.1 .15 & 15.1 .15 & 16.1 .15 & 17.1 .15 & 19.1 .15 & 21.1 .15 & 22.1 .15 & 23.1 .15 & 24.1 .15 \\
\hline INR & 7,88 & 4,63 & 2,36 & 2,31 & 1,69 & 1,59 & 1,31 & 1,3 & 1,18 & 1,15 & 1,1 & 1,1 \\
\hline PTZ & 94,2 & 49,3 & 25,8 & 25,3 & 18,7 & 17,7 & 14,7 & 14,5 & 13,2 & 13,1 & 12,6 & 11,8 \\
\hline APTT & 120 & 82,4 & 60,8 & 48,4 & 40,3 & 29,4 & 30,4 & 36,9 & 31,4 & 30,2 & 28,5 & 28,2 \\
\hline Creatinin (mg/dL) & 10,8 & 8,6 & 7,1 & 5 & 3,8 & 4,9 & 7,2 & 6 & 4,1 & 5,2 & 7,1 & 6 \\
\hline Hgb (g/dL) & 8 & 9,3 & 8,4 & 7,5 & 9,8 & 9,6 & 9,45 & 9,6 & 9,8 & - & 9,6 & - \\
\hline Haemodialysis (Session) & + & + & + & + & - & - & + & + & - & - & + & + \\
\hline FFP (unit) & ++ & ++ & + & + & - & - & - & - & - & - & - & - \\
\hline RBC (unit) & + & - & + & ++ & - & - & - & - & - & - & - & - \\
\hline
\end{tabular}

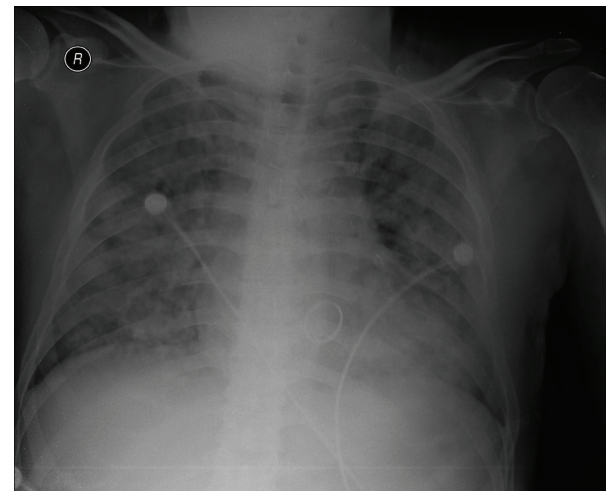

Figure 1: Chest X-ray on admission. Chest X-ray showed alveolar opacities in both lungs, especially in the perihilar and pericardiac zones.

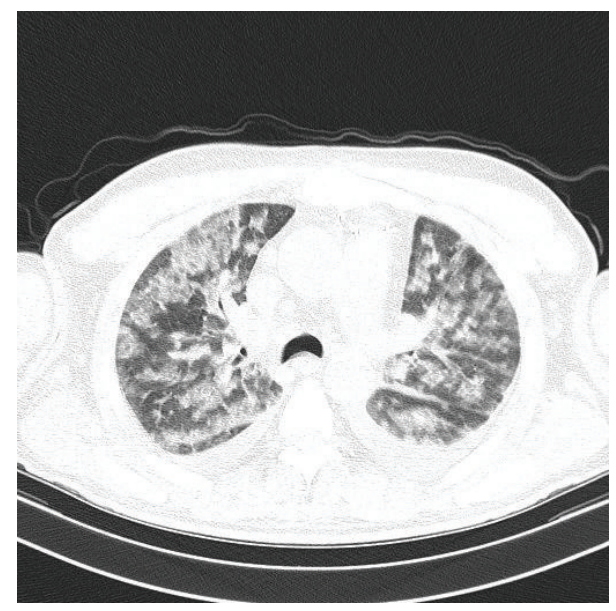

FIGURE 2: Computed tomography of the chest on admission. High resolution CT of the chest showed bilateral patchy airspace disease.

The patient was a known case of CFR due to diabetic nephropathy for the last five years. A double lumen haemodialysis catheter was inserted in his right internal jugular vein as a vascular access for emergency haemodialysis. In total, 8 hemodialysis sessions were carried out during hospital stay. FFP and RBC transfusions were given during hemodialysis sessions to avoid hypervolemia. After FFP administration, coagulopathy improved gradually. When the INR fell below 2, a low molecular weight heparin was started. End-stage renal failure due to diabetic nephropathy was assumed for the patient and he was discharged upon a chronic hemodialysis program (Table 1).

\section{Discussion}

$\mathrm{DAH}$ is a rare occurrence in clinical practice. It can be manifestation of a variety of diseases. The disorders that can cause alveolar hemorrhage involve autoimmune diseases (Wegener granulomatosis, Good Pasteur's syndrome, systemic lupus erythematosus, antiphospholipid antibody syndrome, and Behçet's syndrome), pulmonary infections, cardiac disorders (mitral stenosis), coagulation disorders, bone marrow/solid organ transplantation, toxic exposures, drug reactions (amiodarone, methotrexate, etc.), and idiopathic pulmonary haemosiderosis [1]. A few drugs, including the widely used anticoagulant warfarin, have been documented to cause this potentially lethal condition $[2,3]$.

Warfarin is a vitamin $\mathrm{K}$ antagonist anticoagulant drug and has potential adverse effects. A vitamin $\mathrm{K}$ antagonist (VKA; e.g., warfarin) is recommended in selected patients with metallic prosthetic heart valves to prevent valve thrombosis and thromboembolic events. Warfarin dosing is guided by the use of the international normalized ratio (INR). In patients with a mechanical mitral heart valve, VKA therapy with a target of 3.0 (range 2.5 to 3.5) is suggested [4].

Unfortunately, many factors such as diet, concurrent medication changes, poor compliance, or alcohol consumption may result in unexpected fluctuations in INR levels. The main complication of oral warfarin therapy is bleeding, especially from vital organs such as the brain or lung [5]. Alveolar hemorrhage is difficult to diagnose and has high mortality if the treatment is not being started as soon as possible.

We propose that the patients on anticoagulation therapy require strict monitoring with $\mathrm{PT} / \mathrm{INR}$ to avoid serious bleeding complications related to overanticoagulation.

\section{Conflict of Interests}

The authors declare that there is no conflict of interests regarding the publication of this paper. 


\section{References}

[1] O. C. Ioachimescu and J. K. Stoller, "Diffuse alveolar hemorrhage: diagnosing it and finding the cause," Cleveland Clinic Journal of Medicine, vol. 75, pp. 258, 260, 264-265, 2008.

[2] A. Waness, T. Aldabbagh, and M. Harakati, "Diffuse alveolar haemorrhage secondary to warfarin therapy for atrial fibrillation: a case report and literature review," BMJ Case Reports, 2009.

[3] E. Uysal, E. Çevik, S. Solak, Y. A. Acar, and M. Yalimol, "A life-threatening complication of warfarin therapy in ED: diffuse alveolar hemorrhage," American Journal of Emergency Medicine, vol. 32, no. 6, pp. 690.e3-690.e4, 2014.

[4] R. P. Whitlock, J. C. Sun, S. E. Fremes, F. D. Rubens, and K. H. Teoh, "Antithrombotic and thrombolytic therapy for valvular disease: antithrombotic therapy and prevention of thrombosis, 9th ed: American College of Chest Physicians Evidence-Based Clinical Practice Guidelines," Chest, vol. 141, no. 2, pp. e576Se600S, 2012.

[5] J. Hirsh, V. Fuster, J. Ansell, and J. L. Halperin, "American Heart Association/American College of Cardiology foundation guide to warfarin therapy," Journal of the American College of Cardiology, vol. 41, no. 9, pp. 1633-1652, 2003. 


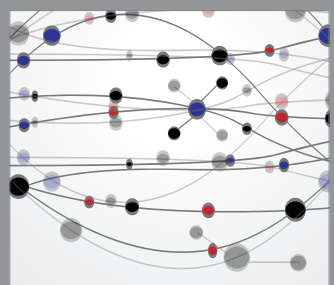

The Scientific World Journal
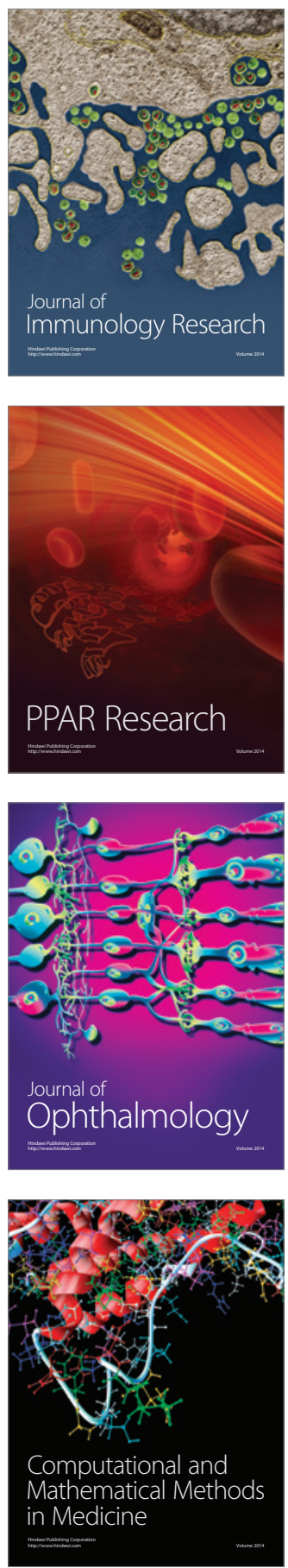

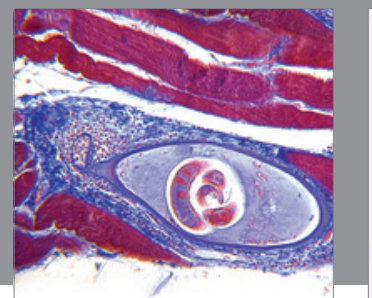

Gastroenterology

Research and Practice
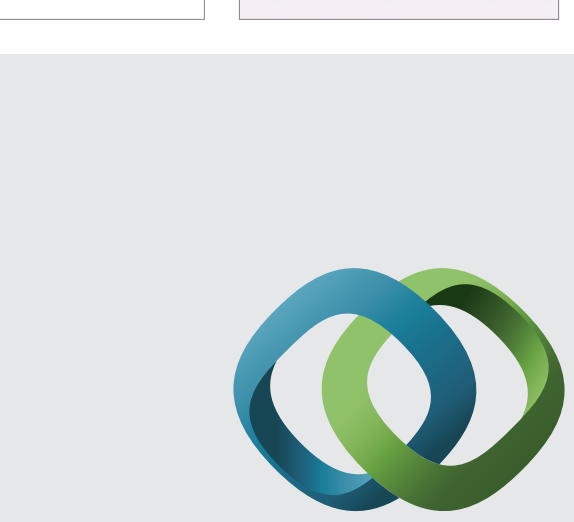

\section{Hindawi}

Submit your manuscripts at

http://www.hindawi.com
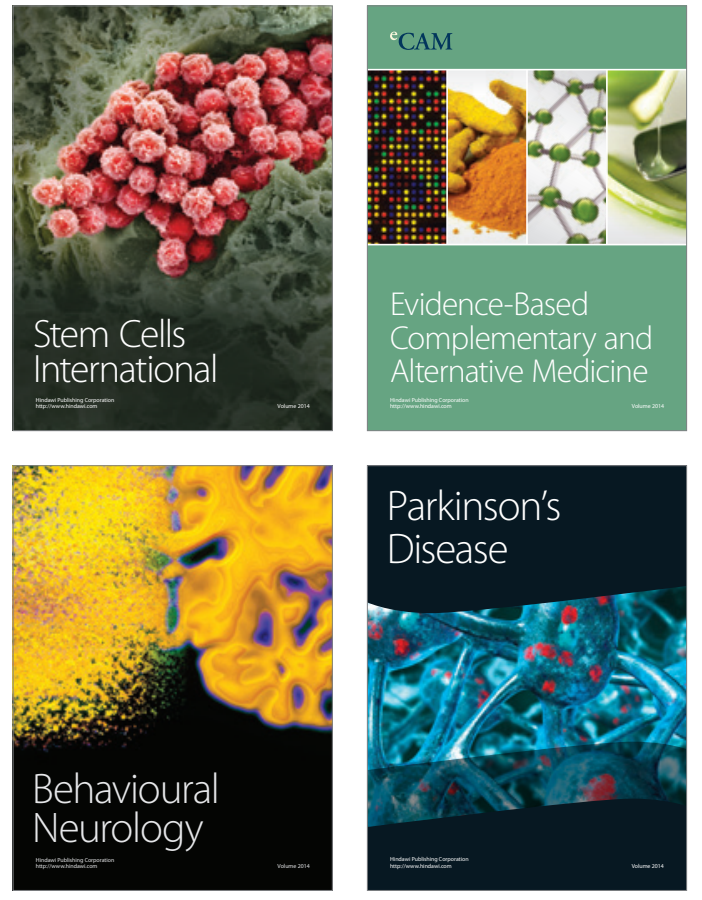
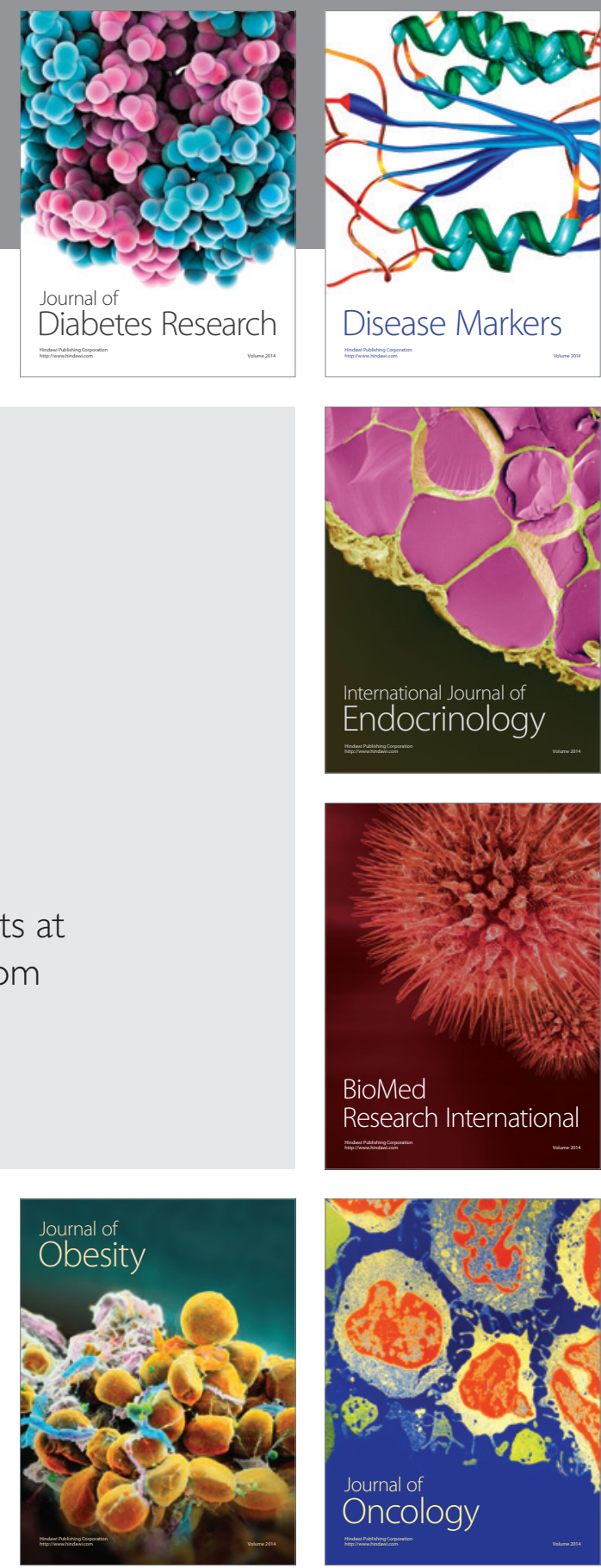

Disease Markers
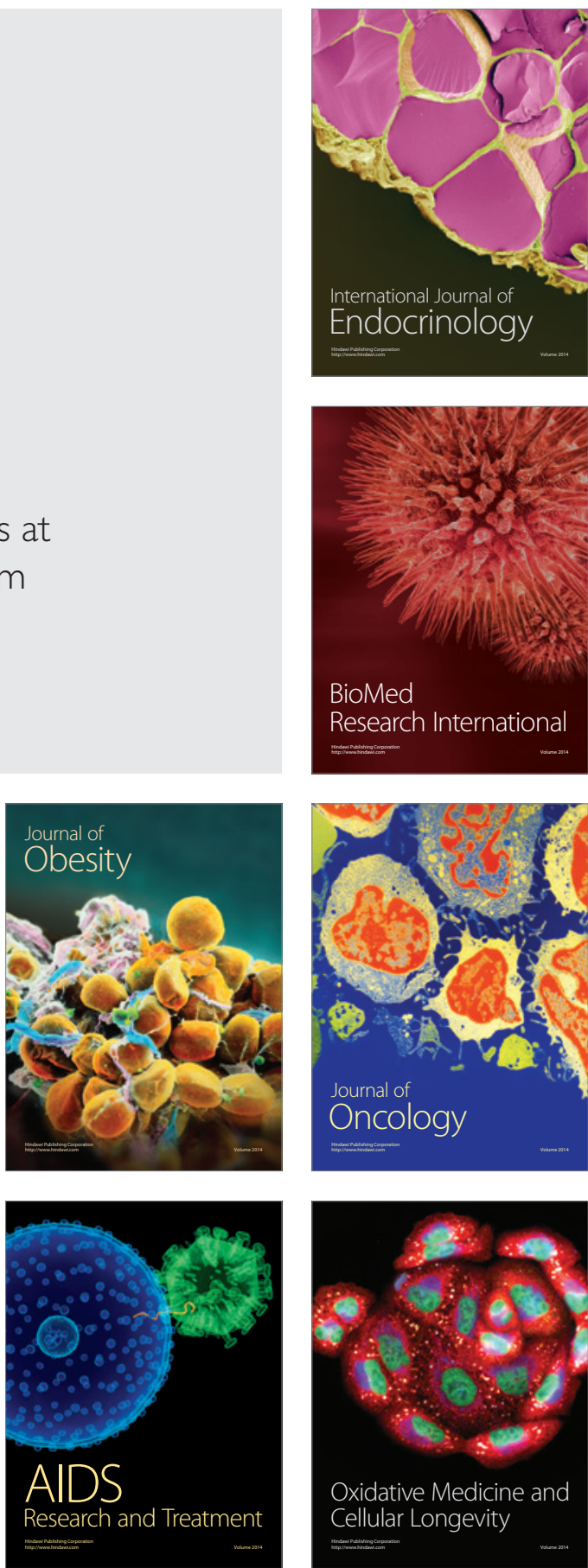